\title{
OCORRÊNCIA DE EIMERIOSE EM BEZERROS CRIADOS EM PROPRIEDADES DE AGRICULTURA FAMILIAR - NOTA CIENTÍFICA
}

\author{
OCCURRENCES OF EIMERIOSIS IN CALVES REARED ON SMALL \\ FAMILY FARMS - SCIENTIFIC REPORT
}

\author{
Leandro Osokoski Hillesheim ${ }^{1 *}$ \\ Fagner Luiz da Costa Freitas ${ }^{1}$ \\ 1Universidade Federal da Fronteira do Sul, Realeza, PR, Brasil \\ *Autor para correspondência - leandrohillesheim@hotmail.com
}

\begin{abstract}
Resumo
A ocorrência de eimeriose foi pesquisada em 110 bezerros da raça holandesa pertencentes a dez propriedades de agricultura familiar. As amostras de fezes foram coletadas via retal e analisadas por métodos parasitológicos qualitativos e quantitativos. Amostras de fezes positivas para Eimeria sp. foram submetidas à esporulação in vitro para posterior identificação microscópica da espécie. Todas as propriedades apresentaram positividade enteroparasitária por espécies do gênero Eimeria havendo uma prevalência de 48,2\% dos animais parasitados. Foram identificadas oito espécies do gênero Eimeria, sendo E. Bovis, E. auburnensis e E. alabamensis as mais frequentes, com $23,6 \%, 11,8 \%$ e 9,1\%, respectivamente. Animais com até seis meses de idade manejados de forma coletiva e a pasto apresentaram maior positividade enteroparasitária. A elevada prevalência de infecção parasitária em animais jovens, principalmente por coccidios de considerável patogenicidade, é indicador de falhas no manejo sanitário, sendo necessário criterioso monitoramento com uso de fármacos específicos para controlar infecções e reduzir perdas zootécnicas.
\end{abstract}

Palavras-chave: Bos taurus; eimeriose; Eimeria bovis.

\begin{abstract}
Occurrences of eimeriosis were investigated among 110 Holstein-Friesian calves belonging to ten small family farms. Feces samples were collected from the rectum and were analyzed using qualitative and quantitative parasitological methods. In vitro sporulation was induced in feces samples positive for Eimeria sp. in order to subsequently identify the species under the microscope. All the farms were positive for species of the genus Eimeria with a prevalence of parasitized animals $48.2 \%$ for Eimeria. Eight species of the genus Eimeria were identified, among which E. bovis, E. auburnensis, and E. alabamensis were the most frequent, accounting for $23.6 \%, 11.8 \%$, and $9.1 \%$, respectively. Animals of up to six months of age that were managed collectively on pasture presented greater positivity for enteroparasites. The high prevalence of parasitic infection in young animals, especially due to coccidia of considerable pathogenicity, is an indicator of failure of sanitary management. Careful monitoring using specific drugs for controlling infections and reducing losses from animal rearing is needed.
\end{abstract}

Keywords: Bos taurus; eimeriosis, Eimeria bovis.

Enviado em: 16 dezembro 2014

Aceito em: 09 outubro 2015 


\section{Introdução}

A eimeriose bovina é uma parasitose causada por protozoários coccídeos do filo Apicomplexa, família Eimeridae, gênero Eimeria. Esta doença afeta, principalmente, animais jovens, com idade compreendida entre quatro semanas e um ano, ocasionando perdas econômicas em todo o mundo ${ }^{(1)}$. O gênero Eimeria é um dos mais importantes protozoários que parasitam o trato gastrintestinal dos bovinos, desencadeando enterite contagiosa que, entre outros problemas, provoca o aparecimento de diarreia $^{(2)}$.

Os bovinos são parasitados por diversas espécies de Eimeria; no entanto, as espécies E. bovis e E. zuernii são consideradas as de maior importância clínica em todo o mundo, ocasionando distúrbios entéricos em bezerros, podendo resultar na morte dos animais doentes. Já as espécies E. auburnensis e E. alabamensis são consideradas moderadamente patogênicas, enquanto as outras espécies são menos patogênicas e raramente são associadas à doença clínica ${ }^{(3)}$.

Coccídeos do gênero Eimeria são considerados monoxenos e estenoxenos, e seu ciclo apresenta uma fase de reprodução assexuada e outra sexuada ${ }^{(3,4)}$. O ciclo de vida possui uma fase interna, que vai desde a ingestão dos oocistos, à multiplicação de estruturas intermediárias e à eliminação de oocistos não esporulados, até uma fase externa, na qual o parasito está no ambiente, local onde esporula ${ }^{(3)}$. Conforme Jolley e Bardsley ${ }^{(5)}$, oocistos infectantes estão presentes no solo, pastagem e fontes de água de praticamente todos os locais habitados por ruminantes. Os oocistos possuem uma grande capacidade de sobrevivência e mantém a capacidade de infecção por semanas ou meses.

Lima $^{(6)}$ aponta que os principais fatores que interferem nas características da eimeriose são: idade, imunidade, fatores relacionados ao parasito, manejo e clima. Em bezerros, a ocorrência de eimeriose é maior na faixa etária compreendida entre três semanas a seis meses de idade; entretanto, a doença pode atingir animais mais velhos quando a densidade populacional for alta, quando a carga de oocistos ingeridos for grande ou quando os animais estiverem submetidos a estresse, doenças concomitantes ou fraca imunidade.

O sistema de criação é um fator que interfere diretamente sobre as características da eimeriose. Em alta densidade populacional, criações intensivas, falta de higiene em bebedouros, cochos e instalações, a doença ocorre com maior frequência e severidade ${ }^{(6,7)}$, assim como em períodos bastante pluviosos, locais úmidos e com higiene precária ${ }^{(3)}$.

A transmissão da eimeriose ocorre através da ingestão de oocistos esporulados, provenientes de animais infectados. A ingestão pode ocorrer pelo alimento ou água contaminados ou pela lambedura de baias e outros animais, atitude que é frequente em bezerros ${ }^{(5)}$.

Dependendo da espécie e da pressão de infecção, a eimeriose pode se apresentar na forma clínica ou subclínica. Quando a infeção ocorre por um grande número de oocistos, principalmente de $E$. bovis e E. zuernii, haverá o desenvolvimento da doença clínica com diarreia geralmente sanguinolenta, desidratação, anorexia, letargia, febre, perda de peso e eventuais mortes ${ }^{(6,8,9)}$. Por outro lado, quando a pressão de infecção é baixa ou o parasitismo se dá por espécies pouco patogênicas, a eimeriose pode manifestar-se de forma subclínica, provocando queda no desempenho produtivo ${ }^{(6)}$.

A associação de Eimeria sp. com helmintos é bastante frequente ${ }^{(10,11)} \mathrm{e}$ tende a aumentar a gravidade do parasitismo, pois os helmintos normalmente ocasionam diminuição do consumo, da capacidade de digestão e absorção dos nutrientes e diarreia ${ }^{(12)}$.

O diagnóstico da eimeriose é realizado com associação do histórico, quadro clínico, epidemiologia e exame parasitológico, sendo este um método eficiente e pouco oneroso ${ }^{(3)}$.

A identificação da espécie encontrada é importante, pois o achado de poucos oocistos de espécies não patogênicas nas fezes não significa que o animal possui eimeriose. O critério mais utilizado para a 
diferenciação de espécies é a avaliação morfométrica dos oocistos esporulados ${ }^{(4)}$.

Devido à carência de dados sobre a eimeriose bovina na bacia leiteira da região sudoeste paranaense o presente trabalho objetivou avaliar a prevalência de espécies do gênero Eimeria e os fatores de risco contribuintes para infecção em bezerros da raça holandesa em propriedades de agricultura familiar no município de Realeza, Estado do Paraná, Brasil.

\section{Material e Métodos}

O presente estudo foi aprovado pela Comissão de Ética no Uso de Animais da Universidade Federal da Fronteira Sul, protocolo nº 23205.001804/2014-92.

No período compreendido entre dezembro de 2013 a agosto de 2014, foram examinados, de forma aleatória, 110 bovinos da raça Holandesa, machos e fêmeas, com faixa etária compreendida entre um a doze meses, provenientes de 10 propriedades rurais leiteiras do município de Realeza, região Sudoeste do Estado do Paraná.

A coleta de fezes foi realizada diretamente do reto dos animais com o auxílio de sacos plásticos limpos, com identificação do número do animal, sexo, idade e a propriedade rural. As amostras foram armazenadas em caixa isotérmica e encaminhadas ao Laboratório de Parasitologia da Universidade Federal da Fronteira Sul, Campus Realeza-PR, para posterior análise laboratorial.

As fezes foram analisadas pelos métodos de Willis ${ }^{(13)}$ e Gordon e Whitlock ${ }^{(14)}$. As amostras positivas para Eimeria sp. tiveram seus oocistos isolados pela técnica de centrífugo-flutuação em solução de $\mathrm{NaCl}$ a $30 \%$ conforme Faust ${ }^{(15)}$. Posteriormente, os oocistos foram depositados em tubos de ensaio contendo solução de dicromato de potássio $\left(\mathrm{K}_{2} \mathrm{Cr}_{2} \mathrm{O}_{7}\right)$ à 2,5\% para ocorrer o processo de esporulação em temperatura ambiente durante sete dias. A observação das estruturas parasitárias foi realizada em microscópio óptico, com auxílio de uma lente de aumento de 400x. A biometria dos oocistos e identificação das espécies do gênero Eimeria foi realizada de acordo com a morfologia dos oocistos, utilizando-se ocular micrométrica.

Para avaliação dos fatores de risco, foram registradas informações referentes ao sistema de criação dos animais nas propriedades. Os resultados dos exames foram confrontados com a faixa etária dos animais, o sistema de manejo e o tipo das instalações.

A análise estatística foi executada pelo programa R-Studio, utilizando-se o teste Qui-Quadrado ${ }^{(16)}$ com correção de Yates a 5\% de significância, para a comparação da prevalência entre as categorias de animais, e o teste $\mathrm{T}$ de Student a 5\% de significância, para a comparação entre valores de OOPG entre as categorias.

\section{Resultados e Discussão}

Todas as propriedades avaliadas apresentaram positividade enteroparasitária por espécies do gênero Eimeria, com uma prevalência de 48,2\% dos animais parasitados. O número médio de oocistos/ grama fezes eliminados pelos animais infectados foi de 921,70 $\pm 2588,39$.

Houve maior prevalência de animais parasitados por espécies de Eimeria sp. na faixa etária compreendida entre um e seis meses, em comparação com sete a doze meses. A média de eliminação de oocistos também foi maior, confirmando que os animais mais jovens são os mais acometidos pela eimeriose $^{(1)}$ (Tabela 1). Acima dos seis meses de idade, os animais tendem a desenvolver imunidade frente o parasitismo e a contagem de oocistos diminui; contudo, estes continuam sendo fonte de infecção para os animais jovens, o que representa um risco para o rebanho. 
Tabela 1. Prevalência e contagem de oocistos de Eimeria diagnosticados em fezes de bezerros da raça holandesa oriundos do município de Realeza, Estado do Paraná, Brasil, 2014, de acordo com a faixa etária

\begin{tabular}{|c|c|c|c|c|}
\hline \multirow{2}{*}{$\begin{array}{l}\text { Faixa Etária } \\
\text { (meses) }\end{array}$} & \multirow{2}{*}{$\begin{array}{l}\text { Número de } \\
\text { amostras }\end{array}$} & \multicolumn{2}{|c|}{ Positivos } & \multirow{2}{*}{$\begin{array}{l}\text { Contagem de oocistos/grama de } \\
\text { fezes }\end{array}$} \\
\hline & & $\mathbf{n}$ & $(\%)$ & \\
\hline $1-6$ & 68 & $34^{a}$ & $50 \%$ & $1350^{A} \pm 3165,00$ \\
\hline $7-12$ & 42 & $19^{b}$ & $45,2 \%$ & $155,26^{\mathrm{B}} \pm 152,65$ \\
\hline
\end{tabular}

Letras minúsculas desiguais na coluna representam diferença significativa a $5 \%$ de significância pelo teste Qui Quadrado. Letras maiúsculas desiguais na coluna representam diferença significativa a $5 \%$ de significância pelo teste $T$ de Student.

Prevalência maior de Eimeria sp. foi encontrada nos animais criados de forma coletiva e com acesso ao pasto (Tabelas 2 e 3). A prevalência de Eimeria sp. na forma de manejo coletivo foi três vezes maior que a forma individualizada e quase quatro vezes maior nos animais criados no pasto em relação à criação em bezerreiro, confirmando a importância do sistema de criação na epidemiologia da eimeriose. Os animais criados no pasto estão mais sujeitos à contaminação através da ingestão de água e pastagens contaminadas com oocistos esporulados.

A doença também ocorre com maior frequência e severidade ${ }^{(6,7)} \mathrm{em}$ alta densidade populacional, criações intensivas, falta de higiene em bebedouros, cochos e instalações. Daugschies e Najdrowski ${ }^{(3)}$ também afirmaram que a ocorrência de eimeriose é maior em períodos bastante pluviosos, em locais úmidos e de higiene precária, pois estes fatores favorecem a sobrevivência dos oocistos.

Tabela 2. Prevalência e contagem de oocistos de Eimeria diagnosticados em fezes de bezerros da raça holandesa oriundos do município de Realeza, Estado do Paraná, Brasil, 2014 , de acordo com o manejo

\begin{tabular}{ccccc}
\multirow{2}{*}{ Manejo } & \multirow{2}{*}{ Número de amostras } & \multicolumn{2}{c}{ Positivos } & \multirow{2}{*}{ Contagem de oocistos/grama de fezes } \\
\cline { 3 - 4 } & $\mathbf{n}$ & $\mathbf{( \% )}$ & \\
\hline Individual & 29 & $5^{\mathrm{a}}$ & $17,2 \%$ & $610^{\mathrm{A}} \pm 629,88$ \\
\hline Coletivo & 81 & $48^{\mathrm{b}}$ & $59,2 \%$ & $954,16^{\mathrm{A}} \pm 2714,28$ \\
\hline
\end{tabular}

Letras minúsculas desiguais na coluna representam diferença significativa a $5 \%$ de significância pelo teste Qui Quadrado. Letras maiúsculas desiguais na coluna representam diferença significativa a $5 \%$ de significância pelo teste $\mathrm{T}$ de Student.

Tabela 3. Prevalência e contagem de oocistos de Eimeria diagnosticados em fezes de bezerros da raça holandesa oriundos do município de Realeza, Estado do Paraná, Brasil, 2014, de acordo com as instalações

\begin{tabular}{ccccc}
\hline \multirow{2}{*}{ Manejo } & \multirow{2}{*}{ Número de amostras } & \multicolumn{2}{c}{ Positivos } & \multirow{2}{*}{ Contagem de oocistos/grama de fezes } \\
\cline { 3 - 4 } & & $\mathbf{n}$ & $\mathbf{( \% )}$ & \\
\hline Bezerreiro & 32 & $5^{\mathrm{a}}$ & $15,6 \%$ & $390^{\mathrm{A}} \pm 411,4$ \\
\hline A pasto & 78 & $48^{\mathrm{b}}$ & $61,5 \%$ & $977,08^{\mathrm{A}} \pm 2713,83$ \\
\hline
\end{tabular}

Letras minúsculas desiguais na coluna representam diferença significativa a $5 \%$ de significância pelo teste Qui Quadrado. Letras maiúsculas desiguais na coluna representam diferença significativa a $5 \%$ de significância pelo teste T de Student. 
Foram identificadas oito espécies do gênero Eimeria, sendo E. Bovis, E. auburnensis e E. alabamensis as mais frequentes. Não foi possível identificar as espécies de Eimeria em sete amostras de fezes, devido ao reduzido número de oocistos (Tabela 4). Animais com faixa etária compreendida entre um e seis meses apresentaram maior diversidade de espécies do gênero Eimeria quando comparados com a faixa etária de sete a doze meses (Tabela 4). A espécie mais encontrada neste estudo, E. bovis $(23,64 \%)$, também foi a mais frequente em estudos realizados no Sul da Bahia $(24,79 \%)$, no Estado de São Paulo $(21,1 \%)$ e na região Sul de Minas Gerais $(23,2 \%)^{(17,18,11)}$, sendo também a espécie mais frequente em vários estudos realizados em outros países ${ }^{(19-23)}$.

As espécies E. bovis e E. zuernii são de alta patogenicidade, representando graves problemas para a saúde dos animais por causarem enterite moderada à grave. Neste estudo, essas duas espécies somadas foram positivas em $25,4 \%$ das amostras analisadas. Já as espécies E. auburnensis e E. alabamensis são consideradas moderadamente patogênicas ${ }^{(3)}$ e, somadas, representaram positividade em $20 \%$ das amostras.

A espécie $E$ bovis, certamente, é a espécie com maior número de estudos e com maior importância clínica em todo o mundo ${ }^{(11,17-23)}$. Animais infectados por esta espécie desenvolvem lesões no intestino grosso, gerando distúrbios na capacidade de absorção da mucosa, promovendo aumento da excreção de sódio, potássio e cloreto $^{(24)}$, além de alterações hematológicas moderadas ${ }^{(3)}$.

Aproximadamente, 4,5\% dos animais examinados foram positivos para E. zuernii, número inferior ao encontrado em vários estudos ${ }^{(11,17,25)}$. Esta espécie apresenta característica singular, possuindo elevada capacidade imunogênica, o que confere imunidade aos bezerros após a primeira infecção ${ }^{(26)}$. Mesmo com a baixa prevalência encontrada, trata-se de uma espécie patogênica que merece atenção, pois ocasiona diarreia sanguinolenta e perda de peso nos bezerros acometidos ${ }^{(29)}$.

Infecções por E. Alabamensis, embora menos patogênicas que E. bovis e E. zuernii, não devem ser ignoradas. Estudos demonstram que em determinados casos esta espécie pode causar doença clínica, embora casos de mortalidade sejam $\operatorname{raros}^{(28,29)}$.

Tabela 4: Frequência de espécies do gênero Eimeria diagnosticadas em fezes de bezerros da raça holandesa oriundos do município de Realeza, Estado do Paraná, Brasil, 2014, de acordo com a faixa etária de um a seis meses $(n=68)$ e sete a doze meses $(n=42)$

\begin{tabular}{|c|c|c|c|c|c|c|}
\hline \multirow{3}{*}{ Espécies } & \multicolumn{6}{|c|}{ Número de Animais Positivos } \\
\hline & \multicolumn{2}{|c|}{ 1-6 Meses } & \multicolumn{2}{|c|}{ 7-12 Meses } & \multicolumn{2}{|c|}{ Total } \\
\hline & $\mathbf{n}$ & $\%$ & $\mathbf{n}$ & $\%$ & $\mathbf{n}$ & $\%$ \\
\hline E. bovis & 18 & 26,47 & 8 & 19,05 & 26 & 23,64 \\
\hline E. auburnensis & 8 & 11,76 & 5 & 11,90 & 13 & 11,82 \\
\hline E. alabamensis & 8 & 11,76 & 2 & 4,76 & 10 & 9,09 \\
\hline E. wyomingensis & 7 & 10,29 & 0 & 0,00 & 7 & 6,36 \\
\hline E. zuernii & 5 & 7,35 & 0 & 0,00 & 5 & 4,55 \\
\hline E. canadenses & 3 & 4,41 & 1 & 2,38 & 4 & 3,64 \\
\hline E. ellipsoidalis & 2 & 2,94 & 0 & 0,00 & 2 & 1,82 \\
\hline E. illinoisensis & 1 & 1,47 & 0 & 0,00 & 1 & 0,91 \\
\hline $\begin{array}{l}\text { Espécie não } \\
\text { identificada }\end{array}$ & 3 & 4,41 & 4 & 9,52 & 7 & 6,36 \\
\hline
\end{tabular}

Os resultados deste estudo são preocupantes, sugerindo que grande parte dos bezerros da região possam estar acometidos por espécies patogênicas de Eimeria. Dessa forma, estes bezerros não têm o aproveitamento ideal dos alimentos ingeridos e as lesões do intestino provocadas pelos parasitos podem ter sequelas para a futura vida produtiva. A diminuição da ingestão de alimentos ${ }^{(30)}$ e a perda de peso $^{(3)}$ provocada pela eimeriose geram um aumento significativo do custo de produção das bezerras, 
que serão à longo prazo a categoria produtiva da propriedade leiteira.

Sabendo da existência do parasitismo por Eimeria spp. na bacia leiteira do Sudoeste Paranaense, animais que desenvolvem diarreia, disenteria, anemia, desidratação, fraqueza, anorexia e caquexia são suspeitos e devem ser examinados para eimeriose ${ }^{(5)}$. Em propriedades com histórico de Eimeria spp., é aconselhável fazer coleta de fezes e monitorar a presença de oocistos. O controle da eimeriose depende muito mais da diminuição da exposição dos animais aos oocistos do que, de fato, da escolha do medicamento. Medidas preventivas incluem espaço apropriado no estábulo, baias limpas, local arejado e chão seco. Jamais bezerros de idades diferentes devem ser misturados e, se algum animal estiver doente, deve ser isolado e tratado.

\section{Conclusões}

Este estudo é o primeiro relato da ocorrência de eimeriose em bovinos criados em propriedades de agricultura familiar da região Sudoeste do Paraná. A elevada prevalência de infecção parasitária em animais jovens, principalmente por coccídios de considerável patogenicidade, é indicador de falhas no manejo sanitário, sendo necessário criterioso monitoramento com uso de fármacos específicos para controlar infecções e reduzir perdas zootécnicas.

\section{Referências}

1. Bruhn FRP, Lopes MA, Demeu FA, Perazza CA, Pedrosa MF, Guimarães AM. Frequency of species of Eimeria in females of the holstein-friesian breed at the post-weaning stage during autumn and winter. Revista brasileira de parasitologia veterinária. 2011 out-dez;20(4):303-7.

2. Rodríguez-Vivas RI, Dominguez-Alpizar JL, Torres-Acosta JF. Epidemiologic factors associated to bovine coccidiosis in calves (Bos indicus) in a subhumid tropical climate. Revista Biomedica. 1996;7(1):211-8.

3. Daugschies A, Najdrowski M. Eimeriosis in catlle: current understanding. Journal of Veterinary Medical Science. 2005;52:417-27.

4. Fayer R. Epidemiology of protozoan infections: the coccidia. Veterinary Parasitology. 1980;6:75-103.

5. Jolley WR, Bardsley KD. Ruminant Coccidiosis. Veterinary Clinics Food Animal Practice. 2006;22:613-21.

6. Lima JD. Coccidiose dos ruminantes domésticos. Revista Brasileira de Parasitologia Veterinária. 2004;13 Suppl 1:9-13.

7. Sánchez RO, Romero JR, Founroge RD. Dynamics of Eimeria oocyst excretion in dairy calves in the Province of Buenos Aires (Argentina), during their first 2 months of age. Veterinary Parasitology. 2008;151:133-8.

8. Stockdale PHG, Bainborought AR, Bailey CB, Niilo L. Some Pathophysiological Changes Associated with Infection of Eimeria zuernii in Calves. Canadian Journal of Comparative Medicine. 1981;45:34-7.

9. Daugschies A, Akimaru M, Burger HJ. Experimentelle Eimeria bovis-Infektionen beim kalb: parasitologische und klinische befunde. Dtsch Tierarztl Wochenschr. 1986;93:393-9.

10. Ferreira MG, Facuri Filho EJ, Heinemann MB, Carvalho AU, Lage AP, Ferreira PM, Freitas MD. Prevalência de Eimeria, helmintos, Escherichia coli, Salmonella, Rotavirus, Coronavirus e Cryptosporidium parvum em propriedades leiteiras de Minas Gerais, Brasil. Ciência Animal Brasileira. 2009; Suppl 1:524-9. 
11. Bruhn, FRP, Silva Júnior FA, Carvalho AHOC, Orlando DR, Rocha CMBM, Guimarães AM. Occurrences of Eimeria spp. and gastrointestinal nematodes in dairy calves in southern Minas Gerais, Brazil. Revista Brasileira de Parasitologia Veterinária. 2012 abr-jun;21(2):171-5.

12. Soutello RVG, Suguisawa L, Cares CCP, Pazeti GCAS, Borges JHR, Brito MNX, Braga CRF, Moraes DAN. Seleção de bovinos de corte resistentes à verminose. Ciências Agrárias e da Saúde. 2002 jul-dez: 2(2): 53-56.

13. Willis HH. A simple levitation method for the detection of hookworm ova. Medical Journal of Australia. 1927;8:375-6.

14. Gordon HMcL, Whitlock HV. A new technique for counting nematode eggs in sheep faeces. Journal Commonwealth Science and Industry Organization. 1939;12(1):50-2.

15. Faust EC, Sawitz W, Tobie J, Odom V, Peres C, Lincome DR. Comparative efficiency of various technics for the diagnosis of protozoa and helminth in feces. The Journal of Parasitology. 1939;(25):241-262.

16. Pearson K. On the criterion that a given system of deviations from the probable in the case of a correlated system of variables is such that it can be reasonably supposed to have arisen from random sampling. Philos Magazine. 1900;5th50:157-175.

17. Rebouças MM, Grasso IMPS, Spósito Filha E, Amaral V, Santos SM, Silva DM. Prevalência e distribuição de protozoários de Gênero Eimeria (Apicomplexa:Eimeridae) em bovinos nos municípios de Altinópolis, Taquaritinga, São Carlos e Guaíra - Estado de São Paulo, Brasil. Revista Brasileira de Parasitologia Veterinária. 1994;3(2):125-130.

18. Almeida VA, Magalhães VCS, Muniz Neta ES, Munhoz AD. Frequency of species of the Genus Eimeria in naturally infected catlle in Southern Bahia, Northeast Brazil. Revista Brasileira de Parasitologia Veterinária. 2011;20(1):78-81.

19. Guardis MV, Brandetti E. Coccidiosis bovina em estabelecimientos tamberos. Identificacion y prevalencia de especies. Revista Medicina Veterinaria. 1986;67(5):282-7.

20. Munyua WK, Ngotho JW. Prevalence of Eimeria Species in Cattle in Kenya. Veterinary Parasitolology. 1990;35:163-8.

21. Cornelissen AW, Verstegen R, Brand H, Perie NM, Eysker M, Lam TJ, Pijpers A. "An observational study of Eimeria species in housed cattle on Dutch dairy farms." Veterinary Parasitology. 1995;56(13):7-16.

22. Faber JE, Kollmann D, Heise A, Bauer C, Failing K, Burger HJ, Zahner H. Eimeria infections in cows in the parturente phase and their calves: oocyst excretion and levels of specific serum and colostrum antibodies. Veterinary Parasitology. 2002;104(1):1-17.

23. Cicek H, Sevimli F, Kozan E, Köse M, Eser M, Dogan N. Prevalence of coccidia in beef cattle in western Turkey. Parasitology Research. 2007;101(5):1239-43.

24. Daugschies A, Bürguer HJ, Akimaru M. Effects of experimental infection with Eimeria bovis on the balance of sodium, potassium and water in calves. Parasitology International. 1997;46:159-69.

25. Bangoura B, Mundt HC, Schmäschke R, Westphal B, Daugschies A. Prevalence of Eimeria ziernii in German Cattle Herds and Factors Influencing Oocist Excretion. Parasitology Research. 2011;109:129-38.

26. Pires P, Capêlo P, Ferreira D, Figueiredo M, Raposo J, Simões J. Prevalência de Eimeria em vitelos clinicamente sãos estabulados em explorações leiteiras. Revista Electrónica de Veterinaria [Internet]. 2013 [cited 2014 Sep 03];14(2):1-12. Available from: http://www.veterinaria.org/revistas/redvet/n020213/021309. pdf Portuguese. 
27. Bangoura B, Daugschies A. Parasitological and clinical parameters of experimental Eimeria zuernii infection in calves and influence on weight gain and haemogram. Parasitology Research. 2007;100:1331-40.

28. Svensson C, Uggla A, Pehrson B. Eimeria alabamensis infection as a cause of diarrhoea in calves at pasture. Veterinary Parasitology. 1994;53: 33-43

29. Samson-Himmelstjerna G, Epe G, Wirtherle N, Heyden V, Weltz C, Radeloff I, Beening J, Carr D, Hellman K, Schnieder T, Krieger K. Clinical and epidemiological characteristics of Eimeria infections in first-year grazind cattle. Veterinary Parasitology. 2006: 136: 215-21.

30. Sartin JL, Shores MA, Schwartz DD, Kemppainen RJ, Baker J. Reduced growth of calves and its reversal by use of anabolic agents. Domestic Animal Endocrinology. 2000;19:85-92. 
